\title{
Quadrotor Control Design under Time and State Constraints: Implicit Lyapunov Function Approach
}

\author{
Siyuan Wang, Andrey Polyakov, Gang Zheng
}

\begin{abstract}
The problem of a state feedback design for control of a quadrotor system under state and time constraints is studied. Convex embedding approach and Implicit Lyapunov function are employed to design a finite-time controller. The feedback gain is solved by a system of LMIs(Linear Matrix Inequalities). Theoretical results are supported with numerical simulation.
\end{abstract}

\section{INTRODUCTION}

Control of quadrotor is an interesting problem that is important for many aeronautical applications. Both linear and nonlinear algorithms are developed for this purpose. For example, algorithms based on PID [1] and LQR design [2], as well as $H_{\infty}$ [3] technique can be classified as linear. The linear design usually uses a system model linearized around an equilibrium point while non-linear terms are omitted. Formally, this means that the linear control is local and an additional robustness analysis is required. As an advance of the linear approach one can be mentioned the simplicity of tuning of control parameters based on Linear Matrix Inequalities (LMIs), which is supported now by MATLAB and many other computational software. In order to have a better performance (e.g. fast maneuvers of the quadrotor), nonlinear controller could be a better solution. A gain scheduling [4] is the simplest way to construct a nonlinear (in fact, a piece-wise linear/switched) control law. The design of a nonlinear controller of quadrotor can be based on feedback linearization [3], backstepping approach [5], [6], sliding mode methodology [7], [8], adaptation [9], [10], and MPC-based (model predictive control) techniques [11], [12].

In practice, the movement of quadrotor is subjected to certain restrictions, e.g. power constraints, computation constraints, state constraints, time constraints and so on. For example, each kind of quadrotor has a limit payload implying that the battery and processing unit cannot be as large as possible. This restricts the endurance time and the computation capacity of the quadrotor. Obviously, simpler controller consumes less energy and responds faster than a complex one. Exceeding some limits of computational power may imply a degradation of control precision. In [13] the authors give estimates showing that a quadrotor controlled by linear

The authors acknowledge the support of CSC Grant and CPER DATA, Project "ControlHub". The second author also acknowledges the support of the Government of Russian Federation (Grant 08-08) and the Ministry of Education and Science of Russian Federation (Project 14.Z50.31.0031)

S. Wang, A. Polyakov and G. Zheng are with Inria Lille-Nord Europe, 40 av. Halley, Villeneuve d'Ascq, 59650, France Siyuan.Wang (Andrey.Polyakov, Gang.Zheng) @inria.fr Polyakov is also with ITMO University, Saint-Petersburg, Russia
PID consumes 5\% less energy than the same quadrotor with a complex (backstepping) controller.

Control of the quadrotor under state constrains is a difficult problem even in some particular cases (see e.g. [14]). In order to satisfy the input, output or state constraints, the control methods such as backstepping [15] or nested saturation [16], [17] could be applied. For linear algorithms an LMI-based schemes can be proposed in order to fulfill state constraints.

Restrictions to transient times (i.e. time constraints) may appear, for example, for the trajectory tracking [18] or the formation control of quadrotors [19] for safety reasons and collision avoidance. Finite-time stabilization [20], is the simplest to fulfill this type of constraints. This paper is devoted to finite-time stabilization of the quadrotor under the state constraints.

The paper is organized as follows: the main notations and definitions are given in the next section. Section III presents the problem statement. Model decomposition and its local convex embedding are considered in Section IV. Section $\mathrm{V}$ designs a controller which allows some time and state constraints to be fulfilled. Section VI and VII present the simulation results and conclusions respectively.

\section{Preliminaries}

\section{A. Notations}

Throughout this paper the following notation will be used:

- $\mathbb{R}$ is the set of real numbers, $\mathbb{R}_{+}=\{x \in \mathbb{R}: x>0\}$.

- $\|\cdot\|$ is the Euclidian norm in $\mathbb{R}^{n}$

- $\operatorname{diag}\left\{\lambda_{i}\right\}_{i=1}^{n}$ is the diagonal matrix with elements $\lambda_{i}$

- $P>0(<0, \geq 0, \leq 0)$ for $P \in \mathbb{R}^{n \times n}$ means that $\mathrm{P}$ is symmetric and positive(negative) definite(semidefinite).

- $\lambda_{\min }(P)$ and $\lambda_{\max }(P)$ represent the minimal and maximal eigenvalue of symmetric matrix $P=P^{T}$ respectively.

- For $P \geq 0$ the square root of $P$ is a matrix $M=P^{\frac{1}{2}}$ such that $M^{2}=P$.

- A continuous function $\sigma: \mathbb{R}_{+} \rightarrow \mathbb{R}_{+}$belongs to the class $\mathcal{K}$ if it is monotone increasing and $\sigma(h) \rightarrow 0^{+}$as $h \rightarrow 0^{+}$.

\section{B. Definitions}

Consider the system of form

$$
\dot{x}=f(t, x), \quad x(0)=x_{0}
$$

where $x \in \mathbb{R}^{n}$ is the state vector, $f: \mathbb{R}_{+} \times \mathbb{R}^{n}$ is a nonlinear vector field.

Definition 2.1: ([21][22][23]) The origin of system is said to be globally finite-time stable if: 
1) Finite-time attractivity: there exists a locally bounded function $\mathfrak{T}: \mathbb{R}^{n} \backslash\{0\} \rightarrow \mathbb{R}_{+}$, such that for all $x_{0} \in$ $\mathbb{R}^{n} \backslash\{0\}$, any solution $x\left(t, x_{0}\right)$ of system is defined at least on $\left[0, \mathfrak{T}\left(x_{0}\right)\right)$ and $\lim _{t \rightarrow \mathfrak{T}\left(x_{0}\right)} x\left(t, x_{0}\right)=0$

2) Lyapunov stability: $\exists \delta \in \mathcal{K}$ such that $\left.\| x\left(t, x_{0}\right)\right) \| \leq$ $\delta\left(\left\|x_{0}\right\|\right)$ for all $x_{0} \in \mathbb{R}^{n}, t \in \mathbb{R}_{+}$

The function $\mathfrak{T}$ is called the settling time function of system.

Theorem 2.1: [21] If there exists a continuous function

$$
\begin{array}{r}
Q: \mathbb{R}_{+} \times \mathbb{R}^{n} \rightarrow \mathbb{R} \\
(V, x) \rightarrow Q(V, x)
\end{array}
$$

satisfying the conditions

C1) $Q$ is continuously differentiable outside the origin;

C2) for any $x \in \mathbb{R}^{n} \backslash\{0\}$ there exists $V \in \mathbb{R}_{+}$such that

$$
Q(V, x)=0
$$

C3) let $\Omega=\left\{(V, x) \in \mathbb{R}_{+} \times \mathbb{R}^{n}: Q(V, x)=0\right\}$ and

$$
\begin{aligned}
& \lim _{x \rightarrow 0,(V, x) \in \Omega} V=0^{+}, \lim _{V \rightarrow 0^{+},(V, x) \in \Omega}\|x\|=0, \\
& \lim _{\|x\| \rightarrow \infty,(V, x) \in \Omega} V=+\infty
\end{aligned}
$$

C4) $\frac{\partial Q(V, x)}{\partial V}<0$ for all $V \in \mathbb{R}_{+}$and $x \in \mathbb{R}^{n} \backslash\{0\}$;

C5) There exist $c>0$ and $0<\mu \leq 1$ such that

$$
\sup _{t \in \mathbb{R}_{+}} \frac{\partial Q(V, x)}{\partial x} f(t, x) \leq c V^{1-\mu} \frac{\partial Q(V, x)}{\partial V}
$$

for all $(V, x) \in \Omega$

then the origin of the system is globally uniformly finite-time stable and $\mathfrak{T}\left(x_{0}\right) \leq \frac{V_{0}^{\mu}}{c \mu}$, where $Q(V, x)=0$.

\section{Problem Statement}

The dynamic model of quadrotor is well established in the literature (see, e.g. [24], [25]). Since the hub forces and moments are very small compared to actuator moments and forces, they are negligible in the considered model [26].

Two different coordinate systems are used in the system model: the initial and body coordinate systems, denoted by $i$ and $b$ respectively (see Fig.1). The position and the attitude in initial frame of quadrotor are given by $(x, y, z)$ and $(\phi, \theta, \psi)$ (roll-pitch-yaw) respectively.

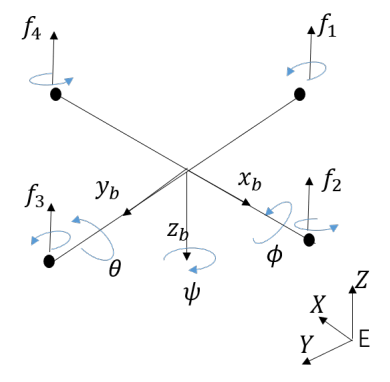

Fig. 1. Quadrotor Coordinate System

The total thrust $F$ of quadrotor in body frame is provided by four thrusts $f_{1}, f_{2}, f_{3}, f_{4}$ of propeller, $F=f_{1}+f_{2}+f_{3}+$ $f_{4}$. The thrust of each propeller is $f_{i}=k \omega_{i}^{2}$, where $k$ is the thrust coefficient and $\omega_{i}$ is the rotation speed of propeller. The force in the inertial frame is given by $R(\phi \theta \psi) F$, where

$$
R(\phi, \theta, \psi)=\left[\begin{array}{ccc}
C_{\theta} C_{\psi} & -S_{\phi} S_{\theta} C_{\psi}+C_{\phi} S_{\psi} & -C_{\phi} S_{\theta} C_{\psi}-S_{\phi} S_{\psi} \\
-C_{\theta} S_{\psi} & S_{\phi} S_{\theta} S_{\psi}+C_{\phi} C_{\psi} & C_{\phi} S_{\theta} S_{\psi}-S_{\phi} C_{\psi} \\
S_{\theta} & S_{\phi} C_{\theta} & C_{\phi} C_{\theta}
\end{array}\right]
$$

with the notations $C_{\theta}=\cos \theta, S_{\theta}=\sin \theta$, etc.

In order to obtain the dynamics equation, the following Newton-Euler equations can be applied:

$$
\left[\begin{array}{cc}
m I_{3 \times 3} & 0 \\
0 & J
\end{array}\right]\left[\begin{array}{c}
\ddot{\xi} \\
\dot{\omega}
\end{array}\right]+\left[\begin{array}{c}
\omega \times m \dot{\xi} \\
\omega \times J \omega
\end{array}\right]=\left[\begin{array}{c}
R(\phi, \theta, \psi) F \\
\tau
\end{array}\right]
$$

Thus we have

$$
\begin{aligned}
m \ddot{\xi}+\left[\begin{array}{c}
0 \\
0 \\
m g
\end{array}\right] & =R(\phi, \theta, \psi) F \\
J \dot{\omega}+\omega \times J \omega & =\tau
\end{aligned}
$$

where $\ddot{\xi}=\left[\begin{array}{lll}\ddot{x} & \ddot{y} & \ddot{z}\end{array}\right]^{T}, \omega=L_{i}^{B} \dot{\Theta}, \mathrm{J}$ is the initia matrix, $\tau$ is the rotation torque with

$$
\begin{aligned}
\dot{\Theta}=\left[\begin{array}{c}
\dot{\phi} \\
\dot{\theta} \\
\dot{\psi}
\end{array}\right], & L_{i}^{B}=\left[\begin{array}{ccc}
1 & 0 & -\sin \theta \\
0 & \cos \phi & \sin \phi \cos \theta \\
0 & -\sin \phi & \cos \phi \cos \theta
\end{array}\right] \\
\tau=\left[\begin{array}{c}
\tau_{\phi} \\
\tau_{\theta} \\
\tau_{\psi}
\end{array}\right], & J=\left[\begin{array}{ccc}
I_{x x} & 0 & 0 \\
0 & I_{y y} & 0 \\
0 & 0 & I_{z z}
\end{array}\right]
\end{aligned}
$$

$m$ the total mass of quadrotor and $\left(I_{x x}, I_{y y}, I_{z z}\right)$ moment of inertia with respect to the axes. The inputs of system are

$$
\left[u_{1}, u_{2}, u_{3}, u_{4}\right]:=\left[F, \tau_{\phi}, \tau_{\theta}, \tau_{\psi}\right]
$$

where

$$
\begin{aligned}
F & =k\left(\omega_{1}^{2}+\omega_{2}^{2}+\omega_{3}^{2}+\omega_{4}^{2}\right) \\
\tau_{\phi} & =\frac{L_{\text {roll }}}{2} k\left(\omega_{1}^{2}-\omega_{3}^{2}\right) \\
\tau_{\theta} & =\frac{L_{\text {pitch }}}{2} k\left(\omega_{2}^{2}-\omega_{4}^{2}\right) \\
\tau_{\psi} & =c\left(-\omega_{1}^{2}+\omega_{2}^{2}-\omega_{3}^{2}+\omega_{4}^{2}\right)
\end{aligned}
$$

with $L_{\text {roll }}$ is the roll motor to motor distance, $L_{\text {pitch }}$ is the pitch motor to motor distance, $c$ is drag coefficient and $k$ is thrust coefficient.

Then the equations of motion in the initial coordinates [24] can be derived from (4), (5) as follows

$$
\begin{aligned}
m \ddot{x} & =(\cos \phi \sin \theta \cos \psi+\sin \phi \sin \psi) u_{1} \\
m \ddot{y} & =(\cos \phi \sin \theta \sin \psi-\sin \phi \cos \psi) u_{1} \\
m \ddot{z} & =\bar{u}_{1} \\
I_{x x} \ddot{\phi} & =\bar{u}_{2} \\
I_{y y} \ddot{\theta} & =\bar{u}_{3} \\
I_{z z} \ddot{\psi} & =\bar{u}_{4}
\end{aligned}
$$


where

$$
\bar{u}=\left[\begin{array}{c}
\bar{u}_{2} \\
\bar{u}_{3} \\
\bar{u}_{1} \\
\bar{u}_{4}
\end{array}\right]=\left[\begin{array}{cccc}
0 & 1 & \frac{I_{x x} \sin \phi \tan \theta}{I_{y y}} & \frac{I_{x x} \cos \phi \tan \theta}{I_{z z}} \\
0 & 0 & \cos \phi & \frac{-I_{y y} \sin \phi}{I_{z z}} \\
1 & 0 & 0 & 0 \\
0 & 0 & \frac{I_{z z} \sin \phi \sec \theta}{I_{y y}} & \cos \phi \sec \theta
\end{array}\right]\left[\begin{array}{c}
u_{1} \cos \phi \cos \theta-m g \\
u_{2}-r_{1} \\
u_{3}-r_{2} \\
u_{4}-r_{3}
\end{array}\right]
$$

and

$$
\mathbf{r}=\left(\begin{array}{l}
r_{1} \\
r_{2} \\
r_{3}
\end{array}\right)=J\left(\frac{\partial L_{i}^{B}}{\partial \phi} \dot{\phi}+\frac{\partial L_{i}^{B}}{\partial \theta} \dot{\theta}\right) \dot{\Theta}+L_{i}^{B} \dot{\Theta} \times\left(J L_{i}^{B} \dot{\Theta}\right)
$$

The quadrotor model (7) has six coordinates $(x, y, z, \phi, \theta, \psi)$ and only four independent inputs. Therefore the quadrotor is an under-actuated system.

Our goal is to design a controller that stabilizes the quadrotor system under the time constraint

$$
\lim _{t \rightarrow \mathfrak{T}\left(\sigma_{0}\right)} \sigma(t)=0, \quad \mathfrak{T}\left(x_{0}\right) \leq \mathfrak{T}_{\max }
$$

and the state constraints:

$$
\begin{aligned}
\sigma_{1}^{2}+\sigma_{2}^{2} & \leq \epsilon_{1,2}^{2}, \\
\sigma_{3}^{2}+\sigma_{4}^{2} & \leq \epsilon_{3,4}^{2}, \\
\left|\sigma_{i}\right| & \leq \epsilon_{i}, \quad i=5, \ldots, 12
\end{aligned}
$$

where

$$
\sigma=(x, y, \dot{x}, \dot{y}, \phi, \theta, z, \psi, \dot{\phi}, \dot{\theta}, \dot{z}, \dot{\psi})^{T}
$$

is the system state, $\mathfrak{T}$ is the settling time function, $\mathfrak{T}_{\max }$ is the time constraint, $\sigma_{0}$ is the initial state of system satisfying the space constraint and the positive constants

$$
\begin{array}{r}
0<\epsilon_{1,2}, \epsilon_{3,4}, \epsilon_{7}, \epsilon_{8}, \epsilon_{9}, \epsilon_{10}, \epsilon_{11}, \epsilon_{12}<+\infty \\
0<\epsilon_{5}, \epsilon_{6}<\frac{\pi}{2} \text { such that } \cos \left(\epsilon_{5}\right) \cos \left(\epsilon_{6}\right) \geq \frac{1}{2}
\end{array}
$$

define the space constraints.

\section{System TRANSFORMATION AND CONVEX EMBEDDING}

For the considered state vector $\sigma$ the system model can be represented in the form

$$
\dot{\sigma}=\bar{A} \sigma+B \bar{u}
$$

where

$$
\begin{aligned}
& \bar{A}=\bar{A}\left(\phi, \theta, \psi, u_{1}\right)=\left(\begin{array}{cccccc}
0 & I & 0 & 0 & 0 & 0 \\
0 & 0 & R E & 0 & 0 & 0 \\
0 & 0 & 0 & 0 & I & 0 \\
0 & 0 & 0 & 0 & 0 & I \\
0 & 0 & 0 & 0 & 0 & 0 \\
0 & 0 & 0 & 0 & 0 & 0
\end{array}\right) \\
& E=E\left(\theta, \phi, u_{1}\right):=\left(\begin{array}{cc}
\frac{\sin \phi u_{1}}{\phi m} & 0 \\
0 & \frac{\sin \theta \cos \phi u_{1}}{\theta m}
\end{array}\right) \text {, } \\
& R=R(\psi):=\left(\begin{array}{cc}
\sin \psi & \cos \psi \\
-\cos \psi & \sin \psi
\end{array}\right) \\
& I=\left(\begin{array}{cc}
1 & 0 \\
0 & 1
\end{array}\right), \quad B=\left(\begin{array}{cc}
0 & 0 \\
0 & 0 \\
0 & 0 \\
0 & 0 \\
\left(\begin{array}{cc}
\frac{1}{I_{x x}} & 0 \\
0 & \frac{1}{I_{y y}}
\end{array}\right) & 0 \\
0 & \left(\begin{array}{cc}
\frac{1}{m} & 0 \\
0 & \frac{1}{I_{z z}}
\end{array}\right)
\end{array}\right)
\end{aligned}
$$

Let us introduce the new variable $\zeta=T \sigma$ where $\mathrm{T}$ is the orthogonal matrix depending on $\psi$ as follows

$$
T=T(\psi):=\left(\begin{array}{cccccc}
R^{-1} & 0 & 0 & 0 & 0 & 0 \\
0 & R^{-1} & 0 & 0 & 0 & 0 \\
0 & 0 & I & 0 & 0 & 0 \\
0 & 0 & 0 & I & 0 & 0 \\
0 & 0 & 0 & 0 & I & 0 \\
0 & 0 & 0 & 0 & 0 & I
\end{array}\right)
$$

Thus the system can be rewritten in the form

$$
\dot{\zeta}=(A+D) \zeta+B \bar{u}, \quad \zeta(0)=\zeta_{0}:=T(\psi(0)) \sigma_{0}
$$

where

$$
A=\left(\begin{array}{cccccc}
0 & I & 0 & 0 & 0 & 0 \\
0 & 0 & E & 0 & 0 & 0 \\
0 & 0 & 0 & 0 & I & 0 \\
0 & 0 & 0 & 0 & 0 & I \\
0 & 0 & 0 & 0 & 0 & 0 \\
0 & 0 & 0 & 0 & 0 & 0
\end{array}\right), \quad D=D(\dot{\psi}):=\dot{T} T^{-1}
$$

Let $e_{i}$ with $1 \leq i \leq 12$ be the identity vector from $\mathbb{R}^{12}$. Lemma 4.1: Let $\Delta \in[0,1]$, the vector

$$
\epsilon=\left(\epsilon_{1,2}, \epsilon_{3,4}, \epsilon_{5}, \epsilon_{6}, \epsilon_{7}, \epsilon_{8}, \epsilon_{9}, \epsilon_{10}, \epsilon_{11}, \epsilon_{12}\right) \in \mathbb{R}_{+}^{10}
$$


satisfies (12) and

$$
A_{i}=\left(\begin{array}{cccccc}
G_{i} & I & 0 & 0 & 0 & 0 \\
0 & G_{i} & E_{i} & 0 & 0 & 0 \\
0 & 0 & 0 & 0 & I & 0 \\
0 & 0 & 0 & 0 & 0 & I \\
0 & 0 & 0 & 0 & 0 & 0 \\
0 & 0 & 0 & 0 & 0 & 0
\end{array}\right), \quad 1 \leq i \leq 8
$$

where

$$
\begin{gathered}
G_{1}=G_{3}=G_{5}=G_{7}=\epsilon_{12}\left(\begin{array}{cc}
0 & 1 \\
-1 & 0
\end{array}\right) \\
G_{2}=G_{4}=G_{6}=G_{8}=-\epsilon_{12}\left(\begin{array}{cc}
0 & 1 \\
-1 & 0
\end{array}\right) \\
E_{1}=E_{2}=g(1+\Delta)\left(\begin{array}{cc}
1 & 0 \\
0 & 1
\end{array}\right) \\
E_{3}=E_{4}=g\left(\begin{array}{cc}
(1+\Delta) & 0 \\
0 & \frac{(1-\Delta) \sin \left(\epsilon_{6}\right) \cos \left(\epsilon_{5}\right)}{\epsilon_{6}}
\end{array}\right) \\
E_{5}=E_{6}=g\left(\begin{array}{cc}
\frac{(1-\Delta) \sin \left(\epsilon_{5}\right)}{\epsilon_{5}} & 0 \\
0 & (1+\Delta)
\end{array}\right) \\
E_{7}=E_{8}=g(1-\Delta)\left(\begin{array}{cc}
\frac{\sin \left(\epsilon_{5}\right)}{\epsilon_{5}} & 0 \\
0 & \frac{\sin \left(\epsilon_{6}\right) \cos \left(\epsilon_{5}\right)}{\epsilon_{6}}
\end{array}\right)
\end{gathered}
$$

Then for any $\sigma \in \mathbb{R}^{12}$ satisfying (11), for any $\lambda \in[0,1]$ and for any $u_{1} \in m g[1-\Delta, 1+\Delta]$ there exist $\alpha_{i} \geq 0$ :

$$
\sum_{i=1}^{8} \alpha_{i}=1 \quad \text { and } \quad \sum_{i=1}^{8} \alpha_{i} A_{i}=A+\lambda D
$$

The latter lemma proves possibility of application of the so-called convex embedding approach(see e.g. [27]) for control design.

\section{Controller Design with Time and state CONSTRAINT}

Introduce the implicit Lyapunov function candidate [21]

$$
Q(V, \zeta):=\zeta^{T} D_{r}\left(V^{-1}\right) P D_{r}\left(V^{-1}\right) \zeta-1
$$

where $V \in \mathbb{R}_{+}, \zeta \in \mathbb{R}^{12}, P \in \mathbb{R}^{12 \times 12}$ is a symmetric positive definite matrix $P>0$ and $D_{r}(\lambda) \in \mathbb{R}^{12 \times 12}$ is a dilation matrix of the form

$$
D_{r}(\lambda)=\left(\begin{array}{cccc}
\lambda^{4} I & 0 & 0 & 0 \\
0 & \lambda^{3} I & \ldots & 0 \\
0 & 0 & \lambda^{2}\left(\begin{array}{ll}
I & 0 \\
0 & I
\end{array}\right) & 0 \\
0 & 0 & 0 & \lambda\left(\begin{array}{ll}
I & 0 \\
0 & I
\end{array}\right)
\end{array}\right), \quad \lambda \in \mathbb{R}_{+}
$$

Denote

$$
H:=\left(\begin{array}{cccccc}
4 I & 0 & 0 & 0 & 0 & 0 \\
0 & 3 I & 0 & 0 & 0 & 0 \\
0 & 0 & 2 I & 0 & 0 & 0 \\
0 & 0 & 0 & 2 I & 0 & 0 \\
0 & 0 & 0 & 0 & I & 0 \\
0 & 0 & 0 & 0 & 0 & I
\end{array}\right)
$$

Theorem 5.1: If for some

$$
\Delta \in\left[\frac{1}{\cos \left(\epsilon_{5}\right) \cos \left(\epsilon_{6}\right)}-1,1\right]
$$

the tuple

$$
(X, Y, \gamma) \in \mathbb{R}^{12 \times 12} \times \mathbb{R}^{4 \times 12} \times \mathbb{R}_{+}
$$

satisfies the system of LMIs

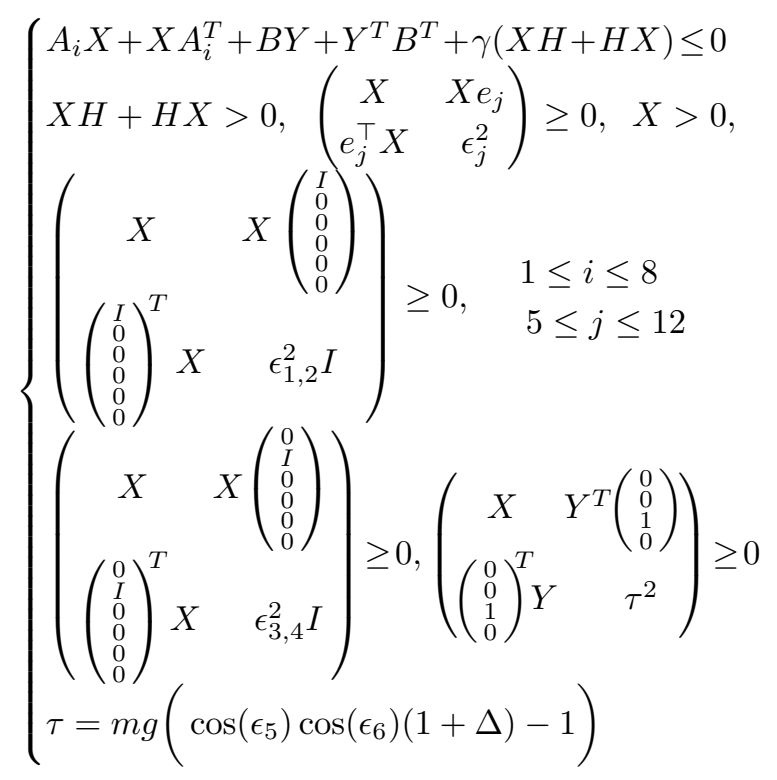

then for any initial condition $\zeta(0)=\zeta_{0}$ satisfying

$$
\zeta_{0}^{T} P \zeta_{0} \leq 1, \quad P=X^{-1}
$$

the controller of the form

$$
\begin{gathered}
\bar{u}=K D_{r}\left(V^{-1}\right) \zeta, \quad K=Y X^{-1} \\
V \in \mathbb{R}_{+}: \zeta^{T} D_{r}\left(V^{-1}\right) P D_{r}\left(V^{-1}\right) \zeta=1,
\end{gathered}
$$

stabilizes the closed-loop system (15) in a finite time

$$
T\left(\zeta_{0}\right) \leq \frac{V\left(\sigma_{0}\right)}{\gamma} \leq \frac{1}{\gamma}
$$

Moreover, the control $\bar{u}$ is globally bounded

$$
\|\bar{u}\|^{2} \leq \lambda_{\max }\left(P^{-\frac{1}{2}} K^{T} K P^{-\frac{1}{2}}\right)
$$


TABLE I

QUADROTOR MODEL PARAMETERS

\begin{tabular}{|c|c|c|c|}
\hline Parameter & Description & Value & Units \\
\hline \hline $\mathrm{g}$ & Gravity & 9.8 & $\mathrm{~m} / \mathrm{s}^{2}$ \\
$\mathrm{~m}$ & Mass & 1.07 & $\mathrm{~kg}$ \\
$L_{\text {roll }}$ & Roll motor distance & 0.2136 & $\mathrm{~m}$ \\
$L_{\text {pitch }}$ & Pitch motor distance & 0.1758 & $\mathrm{~m}$ \\
$I_{x x}$ & Roll Inertia & $6.85 \times 10^{-3}$ & $\mathrm{kgm}^{2}$ \\
$I_{y y}$ & Pitch Inertia & $6.62 \times 10^{-3}$ & $\mathrm{kgm}^{2}$ \\
$I_{z z}$ & Yaw Inertia & $1.29 \times 10^{-2}$ & $\mathrm{kgm}^{2}$ \\
$k$ & Thrust Coefficient & $1.93 \times 10^{-8}$ & $\frac{\mathrm{N}}{R M^{2}}$ \\
$c$ & Drag Coefficient & $0.26 \times 10^{-9}$ & $\frac{N_{m}^{2}}{R P M^{2}}$ \\
\hline
\end{tabular}

and the state constraints (11) are fulfilled for all $t \geq 0$ provided that $\zeta_{0}^{T} P \zeta_{0} \leq 1$.

It is worth stressing that if some states are not constraint, the corresponding LMIs simply disappear for (20).

The parameter $\gamma$ introduced in (20) is for tuning of the settling time. This time can be minimized by means of solving the semi-definite programming problem

$$
\gamma \rightarrow \gamma_{\max }
$$

subject to (20).

\section{SIMULATION RESULTS}

The parameters applied in the simulation is listed in table I.

Suppose that the state constraints are given as

$$
|\dot{\psi}| \leq 1, \quad|\phi| \leq \frac{\pi}{5}, \quad|\theta| \leq \frac{\pi}{5}
$$

Solving LMIs (20) gives the following gain matrix

$K=$

$\left(\begin{array}{cccccccccccc}-62.68 & 0 & -69.31 & 0 & -123.19 & 0 & 0 & 0 & -0.74 & 0 & 0 & 0 \\ 0 & -10.38 & 0 & -119.76 & 0 & -18.36 & 0 & 0 & 0 & -0.98 & 0 & 0 \\ 0 & 0 & 0 & 0 & 0 & 0 & -0.38 & 0 & 0 & 0 & 0 \\ 0 & 0 & 0 & 0 & 0 & 0 & 0 & -19.19 & 0 & 0 & -0.98 & 0 \\ 0 & & & & & 0 & 0 & -33.53\end{array}\right)$

The initial condition here is $\sigma_{0}=$ $[0.24 ;-0.27 ; 0 ; 0 ; 0 ; 0 ; 0.4 ; 0.15 ; 0 ; 0 ; 0 ; 0]$ which makes $\sigma_{0}^{T} P \sigma_{0}=0.958<1$.

Fig. 2 and Fig.3 depict that position and attitude converge to original position in finite time, which means that it converges to zero less than $4 \mathrm{~s}$. The simulation results show that the system converge to zero at around $3 \mathrm{~s}$ for this initial condition. Since the full state of the system is considered together in the controller design, the position and attitude state converge together in the simulation. The constraint of $\dot{\psi}$ are satisfied and confirmed by Fig.4. In the Fig.5, it is clear to see the property of finite-time stability.

The simulation results show that the controller is robust and able to stabilize the quadrotor to the original position under the state and time constrains.

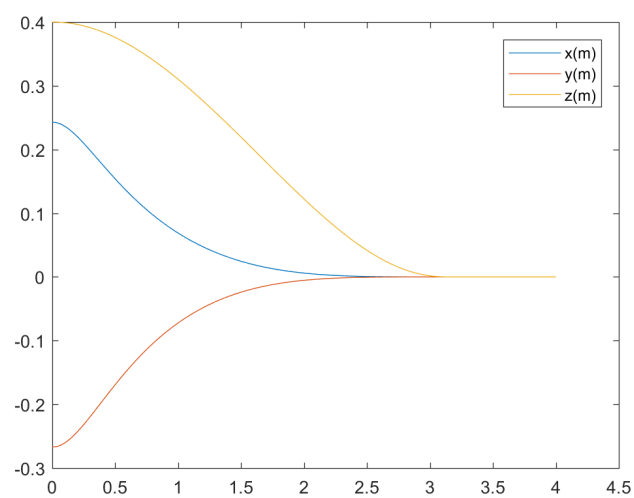

Fig. 2. Quadrotor position $x, y, z$

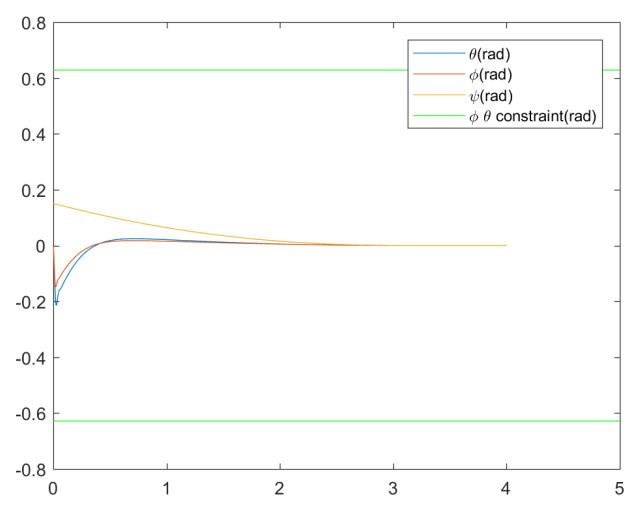

Fig. 3. Quadrotor attitude $\phi, \theta, \psi$

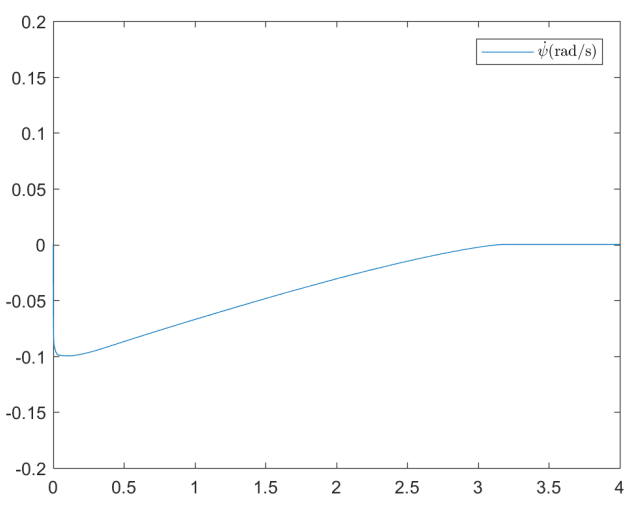

Fig. 4. Angle velocity $\dot{\psi}$ 


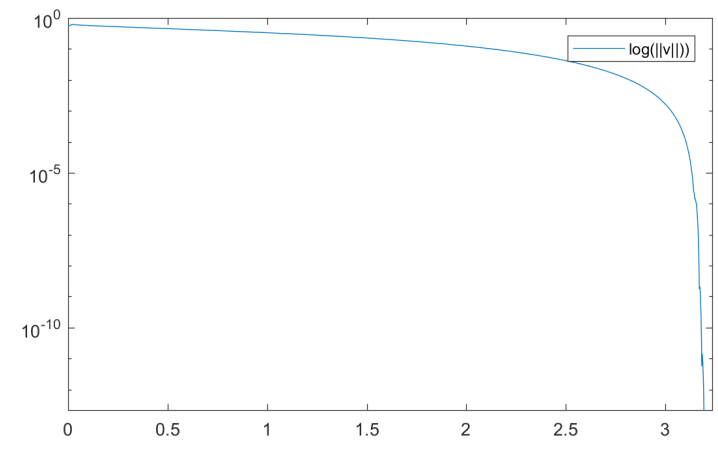

Fig. 5. Log of the norm of vector $v=[x, y, \phi, \theta, z, \psi]$

\section{CONCLUSION}

In this paper the problem of finite-time stabilization of quadrotor under state constraints is studied. A control design scheme based on Implicit Lyapunov function method is proposed. Convex embedding technique is utilized for construction of LMI required for tuning of feedback gains. Control performance is confirmed by numerical simulations.

\section{REFERENCES}

[1] Jun Li and Yuntang Li. Dynamic analysis and pid control for a quadrotor. In 2011 IEEE International Conference on Mechatronics and Automation, pages 573-578. IEEE, 2011.

[2] Samir Bouabdallah, Andre Noth, and Roland Siegwart. Pid vs lq control techniques applied to an indoor micro quadrotor. In 2004 IEEE/RSJ International Conference on Intelligent Robots and Systems (IROS)(IEEE Cat. No. 04CH37566), volume 3, pages 2451-2456. IEEE, 2004.

[3] Abdellah Mokhtari, Abdelaziz Benallegue, and Boubaker Daachi. Robust feedback linearization and $\mathrm{gh} / \mathrm{sub} / \mathrm{spl}$ infin//controller for a quadrotor unmanned aerial vehicle. In 2005 IEEE/RSJ International Conference on Intelligent Robots and Systems, pages 1198-1203. IEEE, 2005.

[4] Ahmad Ataka, Hilton Tnunay, Reka Inovan, Muhammad Abdurrohman, Hanry Preastianto, Adha Imam Cahyadi, and Yoshio Yamamoto. Controllability and observability analysis of the gain scheduling based linearization for uav quadrotor. In 2013 International Conference on Robotics, Biomimetics, Intelligent Computational Systems, pages 212-218. IEEE, 2013.

[5] Samir Bouabdallah and Roland Siegwart. Backstepping and slidingmode techniques applied to an indoor micro quadrotor. In Proceedings of the 2005 IEEE international conference on robotics and automation, pages 2247-2252. IEEE, 2005.

[6] Tarek Madani and Abdelaziz Benallegue. Backstepping control for a quadrotor helicopter. In 2006 IEEE/RSJ International Conference on Intelligent Robots and Systems, pages 3255-3260. IEEE, 2006.

[7] Rong $\mathrm{Xu}$ and Umit Ozguner. Sliding mode control of a quadrotor helicopter. In Proceedings of the 45th IEEE Conference on Decision and Control, pages 4957-4962. IEEE, 2006.

[8] L Luque-Vega, Bernardino Castillo-Toledo, and Alexander G Loukianov. Robust block second order sliding mode control for a quadrotor. Journal of the Franklin Institute, 349(2):719-739, 2012.

[9] S Islam, PX Liu, and A El Saddik. Nonlinear adaptive control for quadrotor flying vehicle. Nonlinear Dynamics, 78(1):117-133, 2014.
[10] Ivana Palunko and Rafael Fierro. Adaptive control of a quadrotor with dynamic changes in the center of gravity. IFAC Proceedings Volumes, 44(1):2626-2631, 2011.

[11] Guilherme V Raffo, Manuel G Ortega, and Francisco R Rubio. Mpc with nonlinear $\mathrm{h}$ infinite control for path tracking of a quad-rotor helicopter. IFAC Proceedings Volumes, 41(2):8564-8569, 2008.

[12] Kostas Alexis, George Nikolakopoulos, and Anthony Tzes. Model predictive quadrotor control: attitude, altitude and position experimental studies. IET Control Theory \& Applications, 6(12):1812-1827, 2012.

[13] Aleksandar Rodić, Gyula Mester, and Ivan Stojković. Qualitative evaluation of flight controller performances for autonomous quadrotors. In Intelligent Systems: Models and Applications, pages 115-134. Springer, 2013.

[14] Najib Metni and Tarek Hamel. A uav for bridge inspection: Visual servoing control law with orientation limits. Automation in construction, 17(1):3-10, 2007.

[15] Mathias Bürger and Martin Guay. A backstepping approach to multivariable robust constraint satisfaction with application to a vtol helicopter. In Proceedings of the 48h IEEE Conference on Decision and Control (CDC) held jointly with 2009 28th Chinese Control Conference, pages 5239-5244. IEEE, 2009.

[16] Ning Cao and Alan F Lynch. Inner-outer loop control for quadrotor uavs with input and state constraints. IEEE Transactions on Control Systems Technology, 24(5):1797-1804, 2016.

[17] Andrew R Teel. Global stabilization and restricted tracking for multiple integrators with bounded controls. Systems \& control letters, 18(3):165-171, 1992.

[18] Bailing Tian, Hanchen Lu, Zongyu Zuo, Qun Zong, and Yunpeng Zhang. Multivariable finite-time output feedback trajectory tracking control of quadrotor helicopters. International Journal of Robust and Nonlinear Control, 28(1):281-295, 2018.

[19] Haibo Du, Wenwu Zhu, Guanghui Wen, and Di Wu. Finite-time formation control for a group of quadrotor aircraft. Aerospace Science and Technology, 69:609-616, 2017.

[20] Arie Levant. Homogeneity approach to high-order sliding mode design. Automatica, 41(5):823-830, 2005.

[21] Andrey Polyakov, Denis Efimov, and Wilfrid Perruquetti. Robust stabilization of mimo systems in finite/fixed time. International Journal of Robust and Nonlinear Control, 26(1):69-90, 2016.

[22] Yury Orlov. Finite time stability and robust control synthesis of uncertain switched systems. SIAM Journal on Control and Optimization, 43(4):1253-1271, 2004.

[23] Emilio Roxin. On finite stability in control systems. Rendiconti del Circolo Matematico di Palermo, 15(3):273-282, 1966.

[24] Samir Bouabdallah. Design and control of quadrotors with application to autonomous flying. Technical report, Epfl, 2007.

[25] Samir Bouabdallah and Roland Siegwart. Full control of a quadrotor. In 2007 IEEE/RSJ International Conference on Intelligent Robots and Systems, pages 153-158. Ieee, 2007.

[26] Pablo SG Cisneros, C Hoffmann, M Bartels, and Herbert Werner. Linear parameter-varying controller design for a nonlinear quad-rotor helicopter model for high speed trajectory tracking. In 2016 American Control Conference (ACC), pages 486-491. IEEE, 2016.

[27] Andrey Polyakov, Laurentiu Hetel, and Christophe Fiter. Relay control design using attractive ellipsoids method. In 2017 IEEE 56th Annual Conference on Decision and Control (CDC), pages 6646-6651. IEEE, 2017. 